\title{
Proposta de um instrumento de enfermagem direcionado à saúde da mulher: revisão integrativa
}

RESUMO | Objetivo: identificar artigos que norteiam a elaboração de uma proposta de instrumento de coleta de dados com base na Teoria das Necessidades Humanas Básicas, como fase inicial do Processo de Enfermagem com o seu guia instrucional. Método: revisão integrativa da literatura, realizada nas bases de dados eletrônicas LILACS, MEDLINE e BDENF disponíveis na BVS/BIREME, no período de 2004 a 2014. Resultados: os sete artigos que corresponderam à amostra do estudo foram indexados na base de dados LILACS, no idioma português, dois foram publicados em 2005, três em 2008, e dois em 2012. Os periódicos foram: Acta Paul Enferm, Esc Ana Nery, Ciênc Cuid Saúde, Rev Eletr Enferm, Rev Bras Enferm, Rev Rene. Conclusão: os artigos analisados chegaram ao mesmo resultado, de que a construção dos instrumentos de coleta de dados de enfermagem foi eficaz para a implantação do PE. O estudo identificou subsídios para estruturar-se de forma científica uma proposta de instrumento que poderá ser aplicado na prática assistencial do enfermeiro.

Palavras-chaves: coleta de dados; anamnese; enfermagem obstétrica.

ABSTRACT | Objective: to identify articles that guide the elaboration of a proposal for a data collection instrument based on the theory of basic human needs, as the initial phase of the Nursing Process with its instructional guide. Method: integrative literature review, conducted in the electronic databases LILACS, MEDLINE and BDENF available in the BVS/BIREME, from 2004 to 2014 . Results: the seven articles that corresponded to the study sample were indexed in the LILACS database, in the Portuguese language, two were published in 2005, three in 2008, and two in 2012The journals were: Acta Paul Enferm, Esc Ana Nery, Ciênc Cuid Saúde, Rev Eletr Enferm, Rev Bras Enferm, Rev Rene. Conclusion: the analyzed articles reached the same result, that the construction of the nursing data collection instruments was effective for the implementation of the NP. The study identified subsidies to scientifically structure a proposal for an instrument that can be applied in nursing care practice.

Keywords: data collection; medical history taking; obstetric nursing.

RESUMEN | Objetivo: identificar artículos que guíen la elaboración de una propuesta de instrumento de recopilación de datos basado en la teoría de las necesidades humanas básicas, como la fase inicial del Proceso de Enfermería con su guía instructiva. Método: revisión de la literatura integrativa, realizada en las bases de datos electrónicas LILACS, MEDLINE y BDENF disponibles en BVS/BIREME, de 2004 a 2014. Resultados: los siete artículos que correspondieron a la muestra de estudio se indexaron en la base de datos LILACS, en portugués, dos se publicaron en 2005, tres en 2008, y dos en 2012. Los periodicos fueron: Acta Paul Enferm, Esc Ana Nery, Ciênc Cuid Saúde, Rev Eletr Enferm, Rev Bras Enferm, Rev Rene. Conclusión: Ios artículos analizados alcanzaron el resultado, que la construcción de los instrumentos de recolección de datos de enfermería fue eficaz para la implementación del PE. El estudio identificó subvenciones para estructurar científicamente una propuesta de instrumento que se puede aplicar en la práctica de cuidados de enfermería.

Descriptores: recolección de datos; anamnesis; enfermería obstétrica.

\section{Maru Jorge de Pinho Barreiros}

Mestre em Enfermagem no Processo do Cuidar em Saúde, Especialista em Administração Hospitalar (UNAERP), Especialista em Linguística Portuguesa (UNIFAP), Enfermeiro Assistencial do Hospital da Mulher Mãe Luzia (HMML), Docente de Ciências Físicas e Biológicas do Ensino Fundamental II, EMEF Maestro Miguel. Macapá (AP), Brasil. Artigo extraído da Dissertação de Mestrado apresentada ao Centro Universitário São Camilo (CUSC). São Paulo (SP), Brasil.

\section{Ivonete Sanches Giacometti Kowalski}

Doutora e Mestre em Educação (Psicologia da Educação) - Pontifícia Universidade Católica de São Paulo (PUCSP). Docente do Curso de Enfermagem do Centro Universitário São Camilo. São Paulo (SP), Brasil.

\section{Grazia Maria Guerra}

Doutora em Ciências (USP), Mestre em Fundamentos de Enfermagem (USP). Pesquisadora do Centro de Pesquisa Clínica Translacional do INCOR - Hospital das Clínicas, USP. Diretora do Departamento de Enfermagem da Sociedade Brasileira de Hipertensão. Coordenadora da Residência da Universidade Santo Amaro (UNISA). São Paulo (SP), Brasil.

Recebido em: 02/06/2019

Aprovado em: 24/10/2019

INTRODUÇÃO

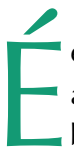
essencial que o enfermeiro possa analisar e avaliar o seu cliente global-mente, tendo como objetivo as dimensões biopsicossociais e espirituais. Quando o corpo e/ou a mente sofrem, a pessoa é afetada em sua totalidade. Portan- to, não podemos dar enfoque somente às partes que perturbam o indívíduo ${ }^{1-2}$. Faz-se necessário que o enfermeiro tenha conhecimento técnico e científico para assistir o seu cliente, resgatando os princípios das necessidades humanas básicas, citadas por Maslow, Mohana e Horta ${ }^{3}$.

Para que essas necessidades sejam atendidas, deve-se realizar uma minuciosa entrevista de enfermagem (anamnese), acompanhado de um completo exame físico, tornando possível um elo de confiança entre o enfermeiro e cliente, visando coletar dados indispensáveis para o seu tratamento e identificar sinais e sintomas da enfermidade ${ }^{3}$.

É importante salientar que a anamnese, hoje chamada de entrevista de enfermagem e o exame físico, caracterizam a fase inicial do Processo de Enfermagem 
$(\mathrm{PE})$, e as demais etapas que compõem este método científico devem ser rigorosamente contempladas. Implantar todas as etapas do PE é uma maneira de tornar a atuação da enfermagem mais científica, possibilitar prognósticos mais precisos $\mathrm{e}$ oferecer uma assistência de enfermagem de qualidade ${ }^{3}$.

A anamnese e o exame físico são peças complementares do PE, que consiste em uma metodologia sistematizada que o enfermeiro põe em prática durante a sua assistência humanizada com o cliente, auxiliando os demais profissionais a tomarem decisões e avaliarem os Diagnósticos de Enfermagem (DE), com a intenção de prevenir complicações e facilitar o tratamento do cliente. Nesta etapa, é feito o levantamento de dados do indivíduo, pois se for insuficiente ou incorreta, pode levar ao DE equivocado, resultando em um planejamento, implementação e avaliação totalmente inadequadas ${ }^{4}$.

$\mathrm{O}$ instrumento de coleta de dados, denominado de Histórico de Enfermagem $(\mathrm{HE})$, pode ser realizado no primeiro contato com o paciente, ou seja, na admissão, podendo ocorrer no domicílio, no ambulatório, no quarto ou na sala de uma clínica ou hospital ${ }^{1}$.

Diante da importância do HE e da inexistência do PE em uma maternidade pública no município de Macapá, surgiu a questão norteadora do estudo: Quais os modelos conceituais utilizados na construção do HE para a avaliação da saúde da mulher, disponíveis na literatura, que contemplam os focos de avaliação de Enfermagem?

A pesquisa tem como meta identificar publicações científicas que norteiam a elaboração de uma proposta de um HE direcionado à saúde da mulher, com base na Teoria das Necessidades Humanas Básicas (TNHB) de Wanda Horta, como fase inicial do PE.

\section{MÉTODO}

Trata-se de uma pesquisa de revisão integrativa da literatura com abordagem quantitativa, utilizada para subsidiar a elaboração de uma proposta de um HE para pacientes em tratamento ginecológico e obstétrico de uma maternidade pública estadual no município de Macapá-AP.

$\mathrm{O}$ estudo percorreu duas principais etapas metodológicas; a primeira foi a revisão integrativa, que seguiu seis procedimentos de busca dos artigos, que são:

a) Seleção dos descritores: a busca e seleção dos dados foram realizadas por meio dos Descritores em Ciências da Saúde (DeCS);

b) Seleção das bases de dados: LILACS, MEDLINE e BDENF, utilizou-se o operador booleano AND entre os descritores, na Biblioteca Virtual em Saúde (BVS/ BIREME);

c) Critérios de inclusão do estudo: artigos publicados nos idiomas português, inglês e espanhol; com texto completo que retratassem a temática referente ao PE com foco no HE, que contemplassem o modelo conceitual de Horta, nas áreas de enfermagem obstétrica e enfermagem ginecológica, no período de 2004 a 2014;

d) Critérios de exclusão do estudo: artigos que não enfatizaram a primeira fase do PE e os focos de avaliação ou atenção de enfermagem, artigos de revisão de literatura e reflexões e artigos replicados;

e) Processo de combinação dos descritores: Coleta de Dados AND Enfermagem Obstétrica; Enfermagem Obstétrica AND Anamnese; Coleta de Dados AND Ginecologia; Processos de Enfermagem AND Exame Físico; Processos de Enfermagem AND Teoria de Enfermagem e Saúde da Mulher AND Registros de Enfermagem;

f) Análise dos artigos na íntegra e motivos de exclusão: foram analisados e selecionados 7 artigos que representam a amostra do estudo. Para a aquisição dos dados e análise dos artigos foi utilizado um instrumento de URSI, validado unicamente para a pesquisa de revisão integrativa da literatura ${ }^{6}$.

A segunda etapa da pesquisa foi a elaboração de uma proposta de HE com o seu guia instrucional. O fluxograma da trajetória metodológica abaixo ilustra as etapas do estudo (Figura 1)..

A elaboração do objeto de estudo deu-se através de dados coletados a partir de três $\mathrm{HE}$, encontrados na amostra, fundamentados pelos Padrões Funcionais de Saúde, Taxonomia II da NANDA e pela Classificação Internacional para as Práticas de Enfermagem (CIPE) Versão 1.0, com

\section{Figura 1. Fluxograma da trajetória metodológica. São Paulo, Brasil, 2019.}

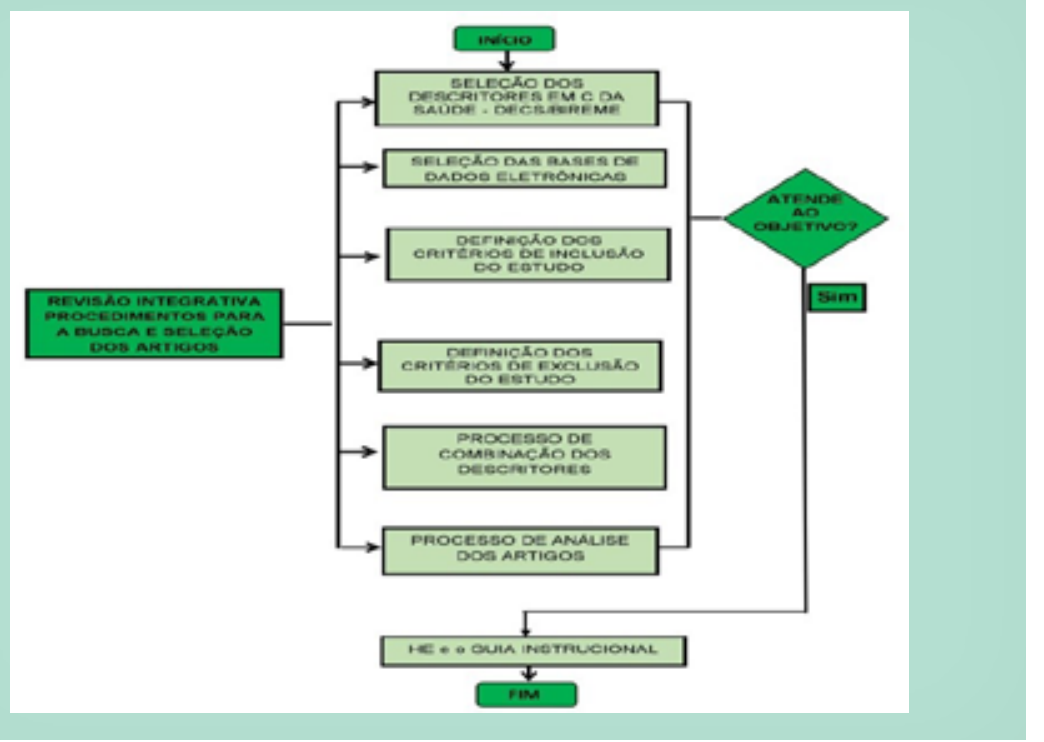


ênfase nos focos de avaliação ou atenção de enfermagem da TNHB, de Horta. Sendo assim, a proposta do HE em formato de checklist foi dividida em duas partes: a identificação e a entrevista de enfermagem e exame físico.

\section{RESULTADOS}

Foram encontradas 285 publicações, sendo 183 artigos, 87 teses, 12 monografias, 1 documento de projeto e 2 documentos não convencionais, com textos completos, incompletos, replicados e indexados nas bases de dados LILACS (263),
MEDLINE (19) e BDENF (3).

Após uma nova busca e seleção dos artigos na BVS/BIREME, chegou-se a um achado de 115 artigos (93,92\%) com texto completo, porém não foram fiéis aos critérios de inclusão propostos pela pesquisa. Em seguida, foi realizada outra busca e seleção minuciosa para que, no mínimo, todos os artigos contemplassem a elaboração e/ou construção do histórico de enfermagem voltado à saúde da mulher.

Apesar dos esforços para a identificação das produções científicas, foi obtido um achado de 7 artigos (6,08\%), que corresponde ao tamanho (n) da amostra desta
Gráfico 1. Distribuição da busca e seleção das produções científicas na BVS/BIREM-E São Paulo, 2019.

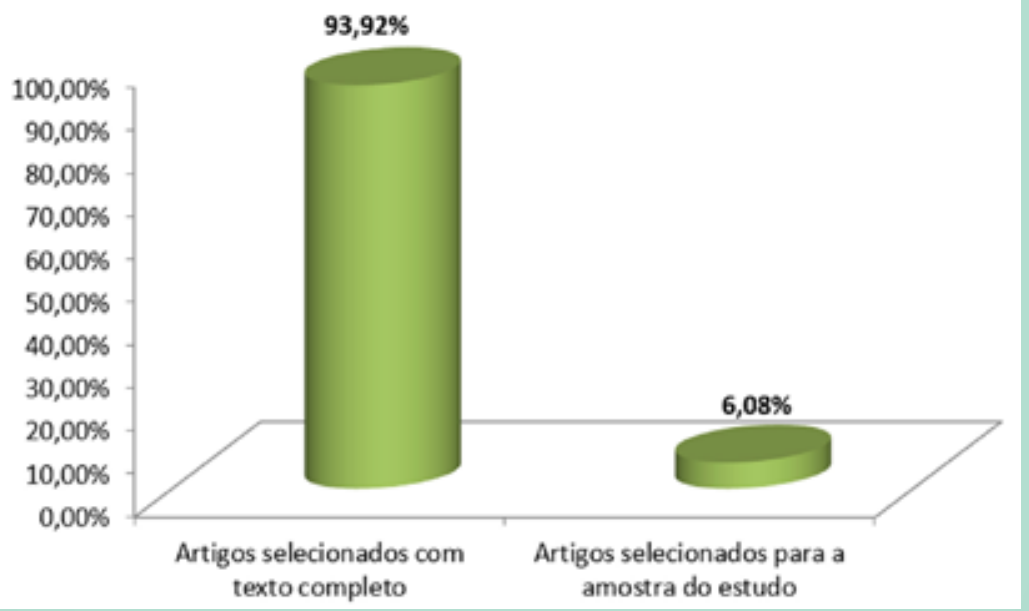

Fonte: o autor, 2019 pesquisa (Gráfico 1).

Todas as publicações científicas referentes à amostra da pesquisa foram encontradas indexadas na base de dados LILACS (100\%), no idioma português (100\%).

Quanto aos periódicos, observou-se uma variedade de revistas, tais como: dois artigos na Acta Paulista de Enfermagem $(28,57 \%)$; um na Escola Ana Nery $(14,28 \%)$; um na Ciência, Cuidado e Saúde (14,28\%); um na Revista Eletrônica de Enfermagem-REE (14,28\%); um na Revista Brasileira de Enfermagem-REBEn (14,28\%) e um artigo na Revista da Rede de Enfermagem do Nordeste-Rev. Rene (14,28\%).

Quanto ao ano de publicação, observou-se que dois artigos foram publicados em $2005(28,57 \%)$, três publicados em 2008 (42,85\%) e dois publicados em $2012(28,57 \%)$.

Com relação ao tipo de publicação, foram encontradas quatro produções científicas no formato de Artigo Original $(57,14 \%)$, duas no de Pesquisa (28,57\%) e uma no formato de Relato de Experiência $(14,28 \%)$. A Caracterização dos artigos estão demonstrados no Quadro 1.

Após a caracterização da amostra do estudo, os artigos foram agrupados de acordo com os modelos conceituais que utilizaram e divididos em três categorias: na Categoria $\mathrm{A}$, foram agrupados 4 artigos $(57,1 \%)$, que fazem abordagem sobre a TNHB e/ou a CIPE nas Versões 1.0 e Beta 2. Na Categoria B, foram agrupados 2 artigos (28,57\%) fundamentados na Taxonomia II da NANDA, com base nos Padrões

\section{Quadro 1 - CARACTERISTICAS DEMOGRÁFICAS DOS PARTICIPANTES}

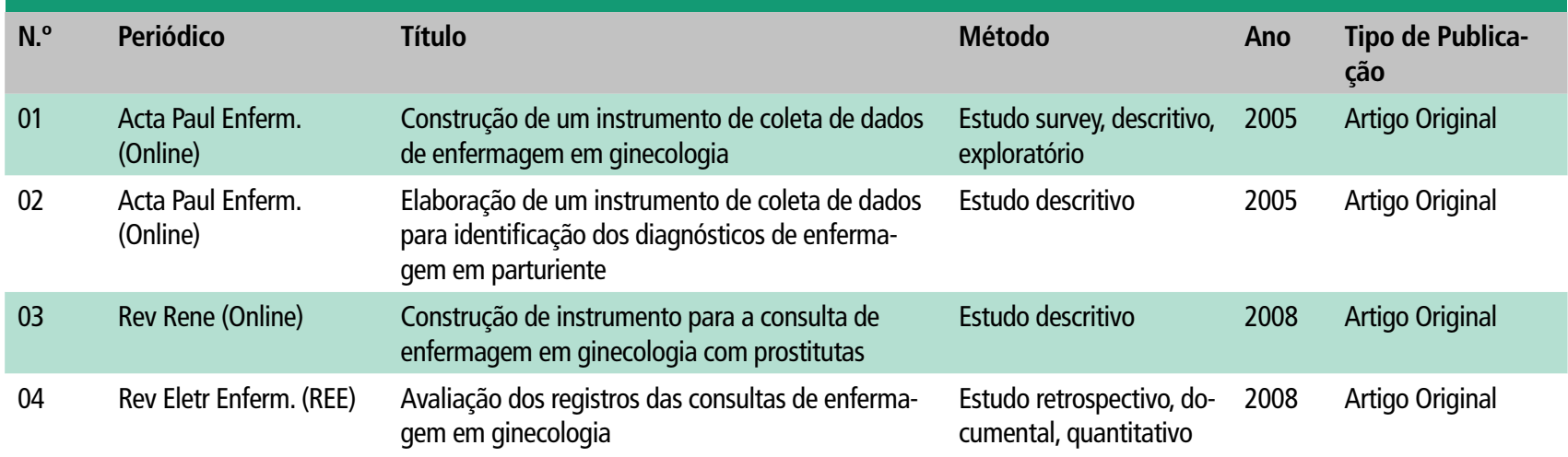




\begin{tabular}{|c|c|c|c|c|c|}
\hline 05 & Ciênc Cuid Saúde. & $\begin{array}{l}\text { Sistematização da assistência em enfermagem } \\
\text { a um binômio mãe-lactentes utilizando a } \\
\text { TNHB e a CIPE@ versão } 1.0\end{array}$ & Estudo de caso & 2008 & $\begin{array}{l}\text { Relato de } \\
\text { Experiência }\end{array}$ \\
\hline 06 & $\begin{array}{l}\text { Rev Bras Enferm. } \\
\text { (REBEn) }\end{array}$ & $\begin{array}{l}\text { Sistematização da assistência de enfermagem } \\
\text { em Centro Obstétrico }\end{array}$ & Revisão de literatura & 2012 & Pesquisa \\
\hline 07 & Esc Anna Nery & $\begin{array}{l}\text { Roteiro de coleta de dados de enfermagem } \\
\text { em alojamento conjunto: contribuições da } \\
\text { articulação ensino-serviço }\end{array}$ & $\begin{array}{l}\text { Pesquisa convergente-assis- } \\
\text { tencial }\end{array}$ & 2012 & Pesquisa \\
\hline
\end{tabular}

Fonte: 0 autor, 2019.

de Saúde Multiaxial e no Modelo de Atividade de Vida. Na Categoria C, foi incluído 1 artigo (14,28\%) que utilizou o Modelo Bifocal da Prática Clínica, segundo os Padrões Funcionais de Saúde.

As informações obtidas foram desmembradas e, de acordo com os modelos conceituais, estabeleceram-se: Teoria das Necessidades Humanas Básicas-TNHB, CIPE Versões 1.0 e Beta 2, Taxonomia II da NANDA, Atividade de Vida e Padrões Funcionais de Saúde (Tabela 1).

\section{DISCUSSÃO}

A enfermagem tem contribuído para pesquisa científica ao longo da história, porém a literatura é escassa no que concerne à saúde da mulher voltada para a primeira fase, o Histórico de Enfermagem do PE, norteado pela TNHB de Horta, visando a demanda de cuidados relacionados aos desequilíbrios do sistema reprodutor feminino e do ciclo vital da reprodução humana em instituições hospitalares.

Com base na análise dos artigos sele-
Tabela 1. Distribuição dos artigos selecionados, de acordo com os modelos teóricos/ conceituais para a elaboração do instrumento. São Paulo, 2019

\begin{tabular}{lcc} 
Modelos teóricos ou conceituais & $\mathbf{N}^{\circ}$ de artigos & $\%$ \\
\hline TNHB & 2 & 22,22 \\
\hline CIPE & 3 & 33,33 \\
\hline Taxonomia II - NANDA & 2 & 22,22 \\
\hline Atividade de Vida & 1 & 11,12 \\
Padrões Funcionais de Saúde & 1 & 11,11 \\
TOTAL & 9 & 100,0
\end{tabular}

Os critérios para a apresentação do HE e do guia instrucional, são:

a) Identificação: inclui o nome da paciente, responsável legal, grau de parentesco, endereço, tempo de residência, data de nascimento, idade, etnia etc.

b) Entrevista de enfermagem e exame físico: faz abordagem às percepções e expectativas a respeito da paciente e às necessidades humanas básicas (NHB): as dimensões psicobiológicas, psicossociais e psicoespirituais. O exame físico está incorporado em cada classe dos referidos domínios do instrumento. cionados, observou-se que a maior parte dos autores utiliza os modelos conceituais: TNHB, NANDA, CIPE, Padrões Funcionais de Saúde e Atividade de Vida concomitantemente, com a finalidade de fortalecer e estabelecer melhor qualidade nos cuidados prestados às pacientes, buscando aperfeiçoar, descrever e construir instrumentos de coleta de dados para tornar a assistência humanizada, com o propósito da implantação ou implementação do PE/ $\mathrm{SAE}$, nas unidades hospitalares.

Santos e Ramos ${ }^{7}$, em um estudo descritivo-exploratório, propuseram um protocolo visando o desenvolvimento da SAE às partu- rientes do centro obstétrico de um hospital público em Recife, baseado na CIPE Versão 1.0 e recomendam que a visão holística do enfermeiro obstetra associado ao PE favoreça uma assistência individualizada.

Souza e colaboradores8, em estudo convergente-assistencial, propõem aperfeiçoar instrumentos de exame físico como primeira fase do $\mathrm{PE}$, embasados na TNHB direcionados à parturiente e ao $\mathrm{RN}$, em alojamento conjunto de uma maternidade de ensino superior, em Belo Horizonte. Evidenciam a importância dessa pesquisa como um método apropriado para introduzir inovações na prática assistencial e a interação entre pesquisadores e enfermeiros.

Albuquerque, Nóbrega e Fontes9 propuseram um estudo de caso, fundamentado na TNHB e na CIPE Versão 1.0, com objetivo de sistematizar a assistência de enfermagem a uma mãe puérpera e suas filhas gêmeas internadas num hospital universitário da Paraíba. Relatam que a aplicação das fases do PE permitiu estabelecer os diagnósticos, resultados e as intervenções de enfermagem, proporcionando melhora na qualidade da assistência.

Para Carvalho e colaboradores10, a consulta de enfermagem ginecológica exige entrevista de enfermagem e exame físico completo. Foi um estudo retrospectivo, documental com abordagem quantitativa, com a finalidade de avaliar os registros das consultas de enfermagem ginecológicas realizadas por acadêmicos de enfermagem no Centro de Parto Natural da Universidade Federal do Ceará. O instrumento utilizado foi um checklist, elaborado conforme requisitos que o Ministério da Saúde preconiza para a consulta em ginecologia, com base na CIPE, Versão Beta 2. 
A pesquisa de Nicolau e colaboradores11 mostra um estudo descritivo sobre a construção de um HE elaborado a partir dos diagnósticos de enfermagem da Taxonomia II da NANDA e norteado pelo Modelo de Atividade de Vida de Roper-Logan-Tierney. Descrevem a construção e aplicação de um HE para a SAE em um serviço de consulta de enfermagem em ginecologia para prostitutas, identificando suas peculiares necessidades.

Sumita, Abrão e Marin12 realizaram um estudo descritivo sobre a construção de um HE para parturiente com base na Taxonomia II da NANDA-Padrões de Saúde Multiaxial. $\mathrm{O}$ instrumento mostrou-se adequado para a coleta de dados em clínica cirúrgica de ginecologia, uma vez que permitiu a identificação de 17 DE, favorecendo a SAE e gerando ações individualizadas.

Já para Soares, Pinelli e Abrão13, em um levantamento descritivo-exploratório, com o objetivo de construir um HE para implantação da SAE numa clínica de cirurgias ginecológicas, fundamentado no Modelo Bifocal da Prática Clínica de Carpenito, segundo os Padrões Funcionais de Saúde. Buscaram uma forma de organizar e registrar um número maior de informa- ções sobre as condições gerais e especiais de saúde das pacientes. O instrumento mostrou-se adequado para a coleta de dados em clínica cirúrgica de ginecologia, pois possibilitou a identificação de $48 \mathrm{DE}$.

Baseado na análise da amostra do estudo, constatou-se que os autores têm a preocupação de tornar o cuidado de enfermagem mais holístico e acolhedor, por meio de instrumentos que subsidiarão as demais etapas do processo como diagnóstico, resultados e intervenções, fundamentados em teorias de enfermagem, escolhidas conforme a realidade assistencial de cada instituição hospitalar. Porém, se o instrumento não obedecer a um raciocínio clínico lógico da coleta de dados, o planejamento assistencial torna-se inoperante.

Todos os estudos selecionados e analisados chegaram ao mesmo resultado, ou seja, que a construção do instrumento foi eficiente e eficaz para a implantação e/ou implementação do PE/SAE. As possíveis lacunas encontradas na amostra foram a inexistência da estrutura formal do HE em seus conteúdos e o não estabelecimento dos critérios de exclusão da população envolvida na pesquisa.

Espera-se que os focos de avaliação contidos no HE deste estudo proporcionem maior clareza para a obtenção de informações importantes das pacientes, podendo facilitar a identificação dos DE para subsidiar ações voltadas ao planejamento da assistência, a fim de descrever os resultados e intervenções de enfermagem específicas e individualizadas, segundo as NHB detectadas. $\mathrm{O}$ instrumento proposto deve passar, em outro momento, por testes, refinamento e validação, antes de ser implantado na prática assistencial pelo enfermeiro na maternidade.

\section{CONCLUSÃO}

O estudo permitiu alcançar o objetivo proposto, o de elaborar uma proposta de instrumento de coleta de dados de enfermagem, com base na teoria das Necessidades Humanas Básicas de Horta, a partir da revisão integrativa da literatura para pacientes em tratamento ginecológico e obstétrico de uma maternidade pública estadual, com a finalidade não só de prestar uma assistência sistematizada, mas também de dar subsídio ao raciocínio ou julgamento clínico do enfermeiro durante a sua prática diária.

\section{Referências}

1. Horta WA. Processo de enfermagem: enfermagem essencial. Rio de Janeiro: Guanabara Koogan; 2011.

2. Maslow AH. Introdução à psicologia do ser. 2. ed. Rio de Janeiro: Eldorado; 1978. 3. Santos N, Veiga P, Andrade R. Importância da anamnese e do exerćício físico para o cuidado ao enfermeiro. Rev Bras Enferm [Internet]. 2011 [citado 2018 ago. 23];64(2):355-8. Disponível em: http://www.scielo.br/pdf/reben/v64n2/a21v64n2.pdf 4. Freitas EP, Nass F, Sponchiado F. 0 processo de enfermagem como perspectiva na melhoria da qualidade da assistência. Rev Enferm [Internet]. 2007 [citado 2019 jan 23];2(2-3):45-52. Disponível em: http://revistas.fw.uri.br/index. php/revistadeenfermagem/article/view/1018/1492

5. Conselho Federal de Enfermagem. Resolução $n^{\circ}$ 358, de 15 de outubro de 2009. Dispõe sobre a Sistematização da Assistência de Enfermagem e a implementação do Processo de Enfermagem em ambientes, públicos ou privados, em que ocorre o cuidado profissional de enfermagem e dá outras providências [Internet]. Diário Oficial da União. 2009 out. 23 [citado 2018 dez 19]. Disponível em: http://www.cofen.gov.br/resoluo-cofen-3582009_4384.html 6. Ursi ES, Galvão CM. Perioperative prevention of skin injury: an integrative literature review. Rev Latinoam Enferm [Internet]. $2006 \mathrm{Jan} /$ Feb [citado 2018 nov 14];14(1):124-31. Disponível em: http://dx.doi.org/10.1590/S010411692006000100017

7. Santos RB, Ramos KS. Sistematização da assistência de enfermagem em Centro Obstétrico. Rev Bras Enferm [Internet]. 2012 [citado 2018 ago 13];65(1):13-8. Disponível em: http://www.scielo.br/pdf/reben/v65n1/02.pdf 8. Souza KV, Assis LTM, Chianca TCM, Ribeiro CL, Gomes AC, Lima RJ. Roteiro de coleta de dados de enfermagem em alojamento conjunto: contribuições da articulação ensino-serviço. Esc Anna Nery [Internet]. 2012 [citado 2018 dez 11];16(2):234-9. Disponível em: http://www.scielo.br/pdf/ean/v16n2/04.pdf 9. Albuquerque CC, Nóbrega MML, Fontes WD. Sistematização da assistência de enfermagem a um binômio mãe-lactentes utilizando a teoria das necessidades humanas básicas e a CIPE@ versão 1.0. Ciênc Cuid Saúde [Internet]. 2008 [citado 2019 mar 15];7(3):392-8. Disponível em: http://www.periodicos. uem.br/ojs/index.php/CiencCuidSaude/article/view/6518/3867 10. Carvalho ALS, Nobre RNS, Leitão NMA, Vasconcelos CTM, Pinheiro AKB. Avaliação dos registros das consultas de enfermagem em ginecologia. Rev Eletr Enferm [Internet]. 2008 [citado 2018 dez 19];10(2):472-83. Disponível em: https://www.fen.ufg.br/revista/v10/n2/pdf/v10n2a18.pdf 11. Nicolau AIO, Aquino PS, Falcão Junior JSP, Pinheiro AKB. Construção de instrumento para a consulta de enfermagem em ginecologia com prostitutas. Rev Rene [Internet]. 2008 [citado 2018 dez 29];9(4):91-8. Disponível em: http://www.periodicos.ufc.br/rene/article/view/5240/3866

12. Sumita SLN, Abrão ACFV, Marin HF. Elaboração de um instrumento de coleta de dados para identificação dos diagnósticos de enfermagem em parturiente. Acta Paul Enferm [Internet]. 2005 [citado 2018 dez 19];18(4):413-21. Disponível em: http://www.scielo.br/pdf/ape/v18n4/a10v18n4.pdf 13. Soares LM, Pinelli FGS, Abrão ACFV. Construção de um instrumento de coleta de dados de enfermagem em ginecologia. Acta Paul Enferm [Internet]. 2005 [citado 2019 fev 10];18(2):156-64. Disponível em: http://www.scielo.br/ pdf/ape/v18n2/a07v18n2.pdf 